Elastic (stress-strain) halo associated with ion-induced nano-tracks in lithium niobate: role of crystal anisotropy

This article has been downloaded from IOPscience. Please scroll down to see the full text article.

2011 J. Phys. D: Appl. Phys. 44475301

(http://iopscience.iop.org/0022-3727/44/47/475301)

View the table of contents for this issue, or go to the journal homepage for more

Download details:

IP Address: 161.111.22.141

The article was downloaded on 11/12/2012 at 11:02

Please note that terms and conditions apply. 


\title{
Elastic (stress-strain) halo associated with ion-induced nano-tracks in lithium niobate: role of crystal anisotropy
}

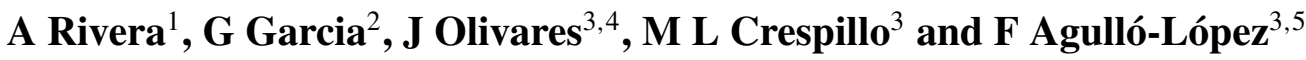 \\ ${ }^{1}$ Instituto de Fusión Nuclear, Universidad Politécnica de Madrid, José Gutiérrez Abascal 2, E28006 \\ Madrid, Spain \\ ${ }^{2}$ ALBA Synchrotron Light Facility, CELLS, E08193 Bellaterra, Barcelona, Spain \\ ${ }^{3}$ Centro de Microanálisis de Materiales, Universidad Autónoma de Madrid, Campus de Cantoblanco, \\ E-28049 Madrid, Spain \\ ${ }^{4}$ Instituto de óptica Daza de Valdés, Consejo Superior de Investigaciones Científicas, E28006 Madrid, \\ Spain \\ ${ }^{5}$ Departamento de Física de Materiales, Universidad Autónoma de Madrid, Campus de Cantoblanco, \\ E-28049-Madrid, Spain \\ E-mail: antonio.rivera.mena@gmail.com
}

Received 21 June 2011, in final form 2 September 2011

Published 4 November 2011

Online at stacks.iop.org/JPhysD/44/475301

\begin{abstract}
The elastic strain/stress fields (halo) around a compressed amorphous nano-track (core) caused by a single high-energy ion impact on $\mathrm{LiNbO}_{3}$ are calculated. A method is developed to approximately account for the effects of crystal anisotropy of $\mathrm{LiNbO}_{3}$ (symmetry $3 m$ ) on the stress fields for tracks oriented along the crystal axes $(X, Y$ or $Z)$. It only considers the zero-order (axial) harmonic contribution to the displacement field in the perpendicular plane and uses effective Poisson moduli for each particular orientation. The anisotropy is relatively small; however, it accounts for some differential features obtained for irradiations along the crystallographic axes $X, Y$ and $Z$. In particular, the irradiation-induced disorder (including halo) and the associated surface swelling appear to be higher for irradiations along the $X$ - or $Y$-axis in comparison with those along the $Z$-axis. Other irradiation effects can be explained by the model, e.g. fracture patterns or the morphology of pores after chemical etching of tracks. Moreover, it offers interesting predictions on the effect of irradiation on lattice parameters.
\end{abstract}

(Some figures may appear in colour only in the online journal)

\section{Introduction}

For swift ions, having specific energies $\sim 1 \mathrm{MeV} \mathrm{amu}^{-1}$, the electronic stopping power, $S_{\mathrm{e}}$, is markedly dominant over the nuclear stopping power, $S_{\mathrm{n}}$, along most of the trajectory. It has now become clear that heavy damage and amorphization can be produced in dielectric and semiconductor crystals by bombarding them with swift ions through electronic excitation mechanisms [1-15]. This electronic excitation damage presents remarkable differential features in comparison with that induced by elastic nuclear collisions and implantation $[7,8,10]$. Experiments on $\mathrm{LiNbO}_{3}$ have shown that the electronic excitation damage is cumulative, as with the nuclear collision damage, but the damage cross-section, $\sigma_{\mathrm{e}}$, is a strongly superlinear function of the electronic stopping power $[8,10]$. Therefore, it reaches much higher values $[8,10]$ than the nuclear collision damage cross-section, $\sigma_{\mathrm{n}}\left(\sigma_{\mathrm{e}} \sim\right.$ $10^{-12} \mathrm{~cm}^{2}$ versus $\sigma_{\mathrm{n}} \sim 10^{-14} \mathrm{~cm}^{2}$ ). Moreover, a welldefined threshold value of $S_{\mathrm{e}}$ is needed to achieve macroscopic amorphization (thresholding effect) in $\mathrm{LiNbO}_{3}[8,10,14]$ and other amorphizable materials [5,11-15]. In fact, it is well known that every single ion generates a well-defined amorphous track of nanometre diameter whenever its stopping power is above such a threshold value. These tracks have been observed and investigated by a variety of techniques [16-29] and offer interesting possibilities for nano-structuring and nano-patterning of materials in electronics and photonics $[11,30-32]$. Although the detailed structure of the tracks is 
not definitely known for most materials and it may be quite complex, it has been, indeed, observed that it includes a central amorphous core surrounded by an extensive halo containing elastically stressed regions as well as point defects (e.g. colour centres) and extended defects [10,25,26]. Understanding the defect structure of the halo requires a definite theoretical model to account for the formation of defects during electronic damage. Recently, we have developed a phenomenological approach for $\mathrm{LiNbO}_{3}$ based on the synergy between the thermal spike concept $[33,34]$ and a non-radiative exciton decay process [35-37]. It allows us to quantify the concentration of point defects generated by swift-ion bombardment, and thus, to estimate the radius of the defective halo and its dependence on electronic stopping power. However, the strain/stress fields associated with the presence of a lower density amorphous track embedded in a denser $\mathrm{LiNbO}_{3}$ crystal host have not, so far, been evaluated.

The purpose of this paper is to evaluate the strain/stress halo around a single amorphous ion track in $\mathrm{LiNbO}_{3}$ and define a meaningful radius as a function of the stopping power of the incident ion. $\mathrm{LiNbO}_{3}$ is a relevant dielectric material where a substantial amount of information on ion-beam damage and track data is available. In a first stage, we summarize the calculation of the displacement and strain fields using isotropic linear elastic theory [38-41] for inclusions embedded in a continuous elastic medium. Our results for the stress/strain halo surrounding the amorphous track core will be compared with those previously obtained for the halo of irradiationinduced point defects, assuming that the two contributions are uncoupled. In a second stage, we have approximately introduced the crystal anisotropy (trigonal $C_{3 m}$ symmetry) in the calculations through the effect of the anisotropic elastic stiffness coefficients. The resulting formalism, despite its simplicity, turns out to be easily applicable to a number of relevant experiments. In fact within this formalism we discuss a number of features associated with the anisotropy and present some new experiments to illustrate our predictions. Moreover, some possible relevant experiments are suggested. The results of this paper are expected to be useful for the application of swift-ion irradiation to nano-patterning in $\mathrm{LiNbO}_{3}$ and other anisotropic materials.

\section{Stress/strain fields (elastic halo) around a single ion track: linear isotropic model}

The problem has been addressed by the Eshelby method $[38,39]$ and by a Green's function approach [40] and it is well documented, see e.g. [41]. Moreover, the elastic analysis has been extended $[42,43]$ to include the role of viscoelastic strains in irradiated amorphous materials (e.g. silica). Here, we will briefly summarize the results corresponding to a cylindrical inclusion in $\mathrm{LiNbO}_{3}$ considered as an infinite elastic continuum isotropic medium with elastic Lamé coefficients $\lambda, \mu$ (or the more practical parameters, Young modulus $E$ and Poisson ratio $v)$. The deviations from isotropy will be analysed in section 3. Let us consider the situation depicted in figure 1, showing an infinitely long amorphous track (inclusion) along the $Z$ direction, having a radius $R$ and the same elastic

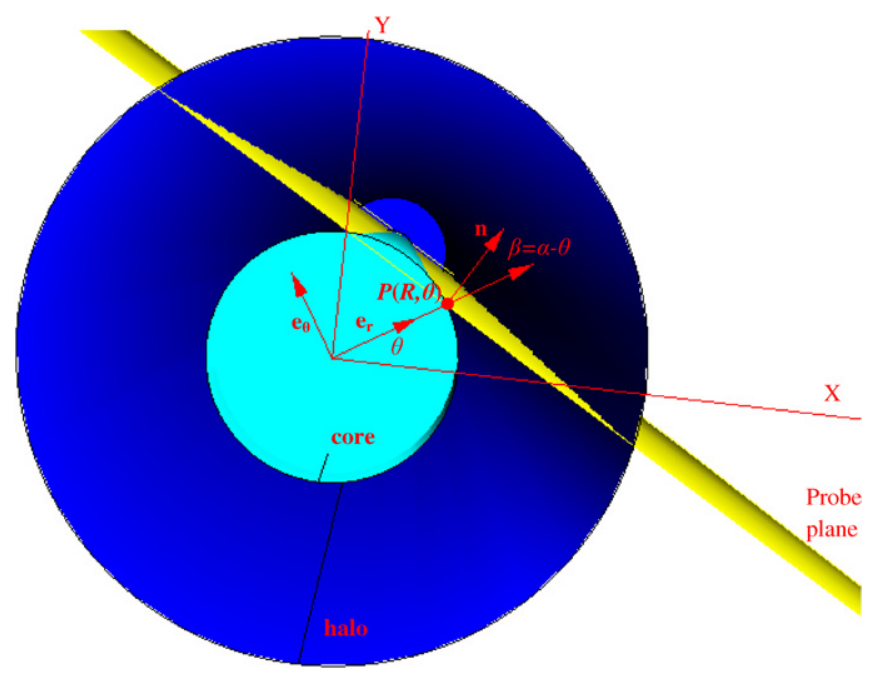

Figure 1. Schematic representation of the $X Y$ cross-section of a track along the $Z$-axis. The amorphous core (of radius $R$ ) and the strained halo are indicated. The normal and shear stresses, $\sigma_{\mathrm{N}}$ and $\sigma_{\mathrm{S}}$, are calculated at a point $P(r, \theta)$ on a probe plane parallel to the track axis $\left(e_{z}\right.$ vector) and whose normal vector $(\boldsymbol{n})$ forms an angle $\beta$ with the $e_{r}$ vector of the cylindrical coordinate basis (equivalently, $\boldsymbol{n}$ forms an angle $\alpha$ with the $X$-axis of the coordinate system).

constants as the crystal host. At any arbitrary point inside the inclusion (amorphous track), i.e. $r<R$, the displacement vector $\boldsymbol{u}$ is radial and strains and stresses are uniform. The most interesting region for our study is the surrounding halo $(r<R)$. For an arbitrary point $P(r, \theta)$ in this region (halo), the displacement vector $\boldsymbol{u}$, the strain tensor $\varepsilon=1 / 2\left(\nabla \boldsymbol{u}+\nabla^{\mathrm{T}} \boldsymbol{u}\right)$ and the stress tensor $\boldsymbol{\sigma}=\lambda \nabla \boldsymbol{u} \boldsymbol{I}+2 \mu \varepsilon$, write

$$
\begin{gathered}
\boldsymbol{u}(r, \theta)=\varepsilon_{0} \frac{1+v}{2(1-v)} \frac{R^{2}}{r} e_{r} \\
\varepsilon(r, \theta)=\varepsilon_{0} \frac{1+v}{2(1-v)} \frac{R^{2}}{r^{2}}\left(\begin{array}{ccc}
-\cos 2 \theta & -\sin 2 \theta & 0 \\
-\sin 2 \theta & \cos 2 \theta & 0 \\
0 & 0 & 0
\end{array}\right)
\end{gathered}
$$

and

$$
\boldsymbol{\sigma}(r, \theta)=\varepsilon_{0} \frac{E}{2(1-v)} \frac{R^{2}}{r^{2}}\left(\begin{array}{ccc}
-\cos 2 \theta & -\sin 2 \theta & 0 \\
-\sin 2 \theta & \cos 2 \theta & 0 \\
0 & 0 & 0
\end{array}\right)
$$

where $\varepsilon_{0}$ (eigenstrain [41]) is the linear uniform expansion at constant pressure caused by the irradiation-induced amorphization of the track core. The above expressions ensure the continuity of the displacement vector and stresses (not the strains) at the boundary. Note that the elastic strains and stresses decrease with the distance from the track axis as $r^{-2}$, i.e. much faster than for a lattice dislocation, as has been experimentally measured for heavy-ion-irradiated zircon [44].

The unit vectors, $\boldsymbol{e}_{\boldsymbol{r}}$ and $\boldsymbol{e}_{\theta}$, together with $\boldsymbol{e}_{\boldsymbol{z}}$, figure 1, define the principal axes of tensors (2) and (3). Therefore, the principal (maximum) strains are

$$
\varepsilon_{r r}=-\varepsilon_{\theta \theta}=-\varepsilon_{0} \frac{1+v}{2(1-v)} \frac{R^{2}}{r^{2}}
$$




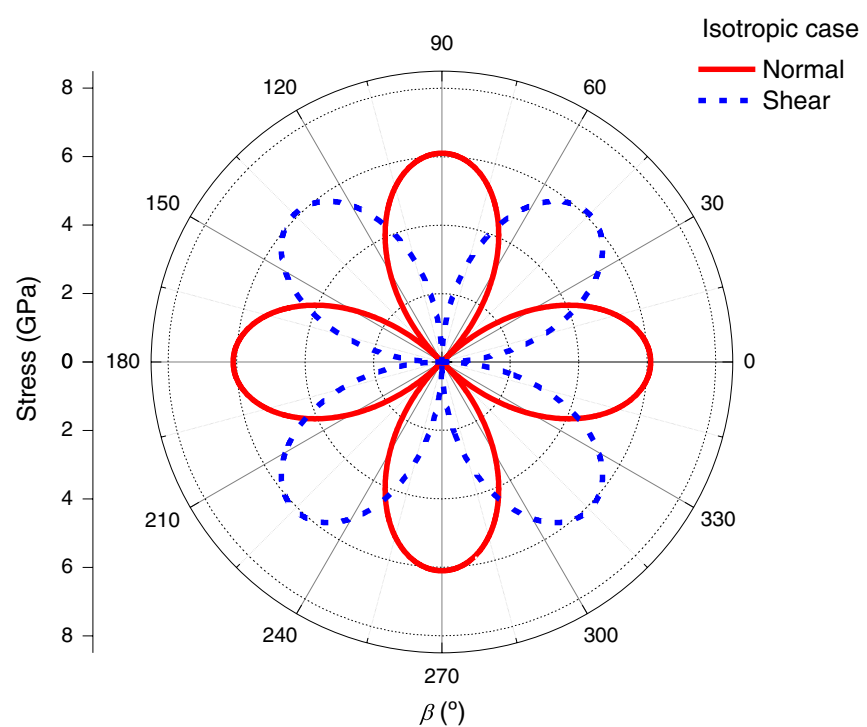

Figure 2. Polar plot for the isotropic case showing the absolute value of the normal and shear stresses at $r=R$ over a probe plane whose normal vector $(\boldsymbol{n})$ forms an angle $\beta$ with the vector $\boldsymbol{e}_{\boldsymbol{r}}$ of the cylindrical coordinate system.

and similarly, the principal (maximum) stresses are

$$
\sigma_{r r}=-\sigma_{\theta \theta}=-\varepsilon_{0} E \frac{1}{2(1-v)} \frac{R^{2}}{r^{2}} .
$$

One may, then, calculate the normal and shear stresses, $\sigma_{\mathrm{N}}(\beta)$ and $\sigma_{\mathrm{S}}(\beta)$, respectively, acting on a probe plane, figure 1 , that contains the $e_{z}$ vector and whose normal vector forms an angle $\beta$ with $\boldsymbol{e}_{\boldsymbol{r}}$ (figure 1):

$$
\begin{aligned}
& \sigma_{\mathrm{N}}(\beta)=\sigma_{r r} \cos 2 \beta \\
& \sigma_{\mathrm{S}}(\beta)=\sigma_{\theta \theta} \sin 2 \beta .
\end{aligned}
$$

\subsection{Strain, stress and elastic energy at the halo}

The absolute values of the $\beta$-angular profiles for $\sigma_{\mathrm{N}}$ and $\sigma_{\mathrm{S}}$ are independent of $\theta$ and are shown in figure 2. The stresses are, obviously, independent of $\theta$ due to the axial symmetry. Absolute values of the normal stresses reach a maximum $\left|\sigma_{r r}\right|=\left|\sigma_{\theta \theta}\right|$ for $\beta=k \pi / 2, k$ being an integer, i.e. for planes perpendicular either to $e_{r}$ or $\boldsymbol{e}_{\theta}$ vectors, and become null for planes forming angles $\beta=k \pi / 4, k$ being an odd integer. The shear stresses behave just in the opposite way, reaching the same maximum value.

In order to obtain some numerical estimates for those components one may use some average value for the anisotropic Poisson coefficients of $\mathrm{LiNbO}_{3}$ (see section 3). One arrives at an average Poisson ratio $v=0.241$ and an average Young modulus $E=186 \mathrm{GPa}$ obtained from coefficients (table 1). Since the linear expansion caused by amorphization is estimated to be around [6] $\varepsilon_{0}=0.05$, one obtains $\varepsilon_{r r}=\left(-\varepsilon_{\theta \theta}\right) \sim-0.04$ for the principal strains at the track core boundary $(r=R)$ and $\sigma_{r r}=\left(-\sigma_{\theta \theta}\right) \sim-6.1 \mathrm{GPa}$ for the principal stresses. These components correspond to the maximum compressive (tensile) strains and stresses at the halo.
Table 1. Elastic stiffness $\left(C_{m n}\right)$ and compliance $\left(S_{m n}\right)$ coefficients for $\mathrm{LiNbO}_{3}$ (from [60]).

\begin{tabular}{lrl}
\hline$m n$ & $\begin{array}{l}C_{m n} \\
(\mathrm{GPa})\end{array}$ & $\begin{array}{l}S_{m n} \\
\left(10^{-14} \mathrm{~m}^{2} \mathrm{~N}^{-1}\right)\end{array}$ \\
\hline 11 & 203 & 578 \\
12 & 53 & -101 \\
13 & 75 & -147 \\
31 & 75 & -147 \\
33 & 245 & 502 \\
14 & 18 & -102 \\
41 & 9 & -51 \\
44 & 120 & 850 \\
66 & 150 & 679 \\
\hline
\end{tabular}

One should mention here that the total elastic (strain) energy associated with the track per unit length is given [41] by

$$
W=\varepsilon_{0}^{2} \frac{E}{1-v} \pi R^{2}
$$

that is proportional to the cross-section of the track core. Therefore, the overall elastic energy density per unit crosssection is essentially independent of the core radius. Using the above quoted average values, $v=0.241, E=186 \mathrm{GPa}$ and $\varepsilon_{0}=0.05$, one obtains an estimate of $W \sim 2 \mathrm{eV} \mathrm{nm}^{-1}$ for unit track cross-section (in $\mathrm{nm}^{2}$ ). This value, which does not include the contribution associated with the phase transition, is very small in comparison with the threshold stopping power for track formation in $\mathrm{LiNbO}_{3}\left(S_{\mathrm{th}} \sim 6 \mathrm{keV} \mathrm{nm}^{-1}\right)$.

\subsection{Radius of the elastic halo}

The existence of an elastic halo around single amorphous tracks formed at stopping powers higher than the threshold has been ascertained or inferred in a few cases by TEM observations [45-47] and from nanopatterns (hillcocks) generated at the emergence of tracks at the sample surface [48-50]. Average information on the lattice distortions caused by multiple-track irradiation can be obtained from highresolution x-ray diffraction experiments $[47,51]$ and RBS/C channelling techniques $[2,24,52]$, although the data cannot usually distinguish between elastic and defective distortions. Regardless, the size of the elastic halo depends on the particular technique used for its detection and its definition is, indeed, a matter of convention. The analysis presented in this paper makes it possible to define an outer radius $R_{\mathrm{H}}$ for the elastic halo surrounding a track core having a radius $R_{\mathrm{C}}=R$. One may consider the elastic halo as the region where the elastic strains (2) induce distortions of the lattice cell that are larger than the average random static or dynamic (vibrational) distortions $\left\langle u_{0}\right\rangle$. Therefore, $R_{\mathrm{H}}$ should be defined through the relation

$$
\varepsilon_{0} \frac{1+v}{2(1-v)} \frac{R^{2}}{R_{\mathrm{H}}^{2}}=\frac{\left\langle u_{0}\right\rangle}{l_{0}}
$$

i.e.

$$
R_{\mathrm{H}}=R \sqrt{\frac{\varepsilon_{0} l_{0}}{\left\langle u_{0}\right\rangle} \frac{1+v}{2(1-v)}}
$$

where $l_{0}$ is a suitable lattice parameter corresponding to the width of a given crystal channel used to probe the crystal 
perfection. The radius of the elastic halo increases linearly with $R$ and with decreasing $\left\langle u_{0}\right\rangle$, i.e. increasing perfection of the crystal. In the ideal case of a perfect crystal, $\left\langle u_{0}\right\rangle$ corresponds to the vibrational amplitude $\left\langle u_{\mathrm{th}}\right\rangle$ and can be roughly inferred from the minimum yield in RBS/C experiments [53]. At room temperature the following roughly isotropic values were obtained, $\left\langle u_{\mathrm{th}}\right\rangle=0.09 \AA$ for $\mathrm{Nb},\left\langle u_{\mathrm{th}}\right\rangle=0.12 \AA$ for $\mathrm{O}$ and $\left\langle u_{\text {th }}\right\rangle=0.15 \AA$ for Li. Considering the dominant channelling role of $\mathrm{Nb}^{5+}$ ions and that the vibration of those atoms constitute the main source for de-channelling (neglecting static distortions), it comes out that $R_{\mathrm{H}} \sim 1.5 \times R$, i.e. the elastic halo is larger than (although comparable to) the amorphous core. Although systematic data are not available this prediction appears consistent with some reports [54]. Moreover, the diameter of the elastic halo may become comparable to that of point defects (defective halo) which makes their experimental separation difficult (see the next section).

\subsection{Comparison of the elastic halo with the surrounding halo of point defects (defective halo)}

In addition to the elastic halo discussed so far, one has to consider the defective region constituted by point defects that are generated by the ion impacts during irradiation and that have not collapsed into the track core. Direct evidence for this surrounding halo is scarce, although it is clear for some materials, such as alkali halides [55] and polymers [56]. Quite relevant to this problem is a recent paper [57] reporting molecular dynamics simulations of swift-ion damage to zircon. The calculations clearly show that the structure of the tracks is complex and consists of an amorphous core surrounded by a halo of point defects. On the other hand, there is abundant kinetics information that provides indirect evidence for the existence of the defective halo and that cannot be applied to the elastic halo. In particular, the Avrami-type kinetics of amorphization by swift ions, for $\mathrm{LiNbO}_{3}$ and other dielectrics and semiconductors, shows an incubation fluence region for low enough stopping powers, which is clearly indicative $[24,58]$ of amorphization as a result of irradiationinduced defect accumulation (cumulative mechanisms).

For $\mathrm{LiNbO}_{3}$, direct quantitative RBS/C data on the size of the defective halo are not very reliable since the de-channelling effect of the halo is quite small in comparison with that of the amorphous core [9]. However, slightly above the threshold, the halo area may represent around $10-20 \%$ of the core area and below the threshold (although the amorphous core is not formed) a defective region (defective halo) is still clearly detectable. Anyhow, one can rely on the cumulative character of defect formation and use the Avrami kinetic laws to make quantitative estimates [59] of the relative sizes of amorphous core and defective halo. The two contributions appear, indeed, comparable for moderate stopping powers. Note that our analysis in section 2.2, together with the results of [59], suggests that for high enough stopping powers the elastic halo should be dominant.

\section{Formulation of the anisotropic stress/strain problem for tracks in $\mathrm{LiNbO}_{3}$}

In order to take into account anisotropy we have to modify the previous isotropic approach using the appropriate anisotropic elastic constants. For $\mathrm{LiNbO}_{3}$ the symmetry is very approximately $C_{3 m}(3 m)$ and the crystal structure is illustrated in figure 3. Note the projection on the $X Y$ plane where the trigonal symmetry is clearly apparent and that the $X$ axis is taken perpendicular to the mirror plane $m$. The true structure involves a small rotation of the octahedra that turns the symmetry into $C_{3}$. However, this effect is of little relevance and it is not usually considered in most structural analyses. The stiffness tensor $\left(C_{i j k l}\right)$ and the compliance tensor $\left(S_{i j k l}\right)$ can be written in a contracted notation ${ }^{6}$ through a $6 \times 6$ matrix with the following form [60]:

$$
Q_{m n}=\left(\begin{array}{cccccc}
Q_{11} & Q_{12} & Q_{13} & Q_{14} & 0 & 0 \\
Q_{12} & Q_{11} & Q_{13} & -Q_{14} & 0 & 0 \\
Q_{31} & Q_{31} & Q_{33} & 0 & 0 & 0 \\
Q_{41} & -Q_{41} & 0 & Q_{44} & 0 & 0 \\
0 & 0 & 0 & 0 & Q_{44} & 2 Q_{41} \\
0 & 0 & 0 & 0 & Q_{14} & Q_{66}
\end{array}\right)
$$

where $Q_{m n}$ represents either the contracted stiffness matrix $\left(C_{m n}\right)$ or the contracted compliance matrix $\left(S_{m n}\right)$. The components fulfil $Q_{66}=Q_{11}-Q_{12}$ and it is usually assumed that $Q_{13} \approx Q_{31}$ and $Q_{14} \approx 2 Q_{41}$. The experimental values [60] of these components at constant field are listed in table 1. Using the above contracted notation the linear relation between stress and strain tensors (also written in contracted notation) becomes

$$
\begin{aligned}
\sigma_{m} & =C_{m n} \varepsilon_{n} \\
\varepsilon_{m} & =S_{m n} \sigma_{n}
\end{aligned}
$$

the latter relation to be used instead of the isotropic relation

$$
\varepsilon_{i j}=\frac{1+v}{E}\left(\sigma_{i j}-\frac{v}{1+v} \sigma_{k k} \delta_{i j}\right) .
$$

A rigorous approach to the anisotropic problem involves solving the general (anisotropic) equilibrium elastic equation instead of the isotropic version and the general formal solution is available [41]. Here, we are interested in the stress/strain profiles associated with amorphous tracks produced by single ion impacts impinging on the crystal along one of the crystal axes $X, Y$ or $Z$. This is the experimental situation most commonly found in practice, when irradiating $X, Y$ or $Z$ crystal cuts at normal incidence. For these cases we will

6 We use the following rules to write the tensors $Q_{i j k l}$ in a contracted notation by means of the $(6 \times 6)$ matrices $Q_{m n}$ :

$\begin{array}{rlrl}\boldsymbol{Q}_{i j k l} & \rightarrow \boldsymbol{Q}_{m n} & \text { with } & \boldsymbol{i j} \rightarrow \boldsymbol{m} \\ Q_{i j 11} & \rightarrow Q_{m 1} & 11 \rightarrow 1 \\ Q_{i j 22} & \rightarrow Q_{m 2} & 22 \rightarrow 2 \\ Q_{i j 33} & \rightarrow Q_{m 3} & 33 \rightarrow 3 \\ Q_{i j 23}+Q_{i j 32} & \rightarrow Q_{m 4} & 23 \text { or } 32 \rightarrow 4 \\ Q_{i j 13}+Q_{i j 31} \rightarrow Q_{m 5} & 13 \text { or } 31 \rightarrow 5 \\ Q_{i j 12}+Q_{i j 21} \rightarrow Q_{m 6} & 12 \text { or } 21 \rightarrow 6\end{array}$

The rules shown in the right column are also used to write the stress $\left(\sigma_{i j}\right)$ and strain $\left(\varepsilon_{i j}\right)$ tensors in a contracted notation by means of the $(6 \times 1)$ vectors $\sigma_{m}$ and $\varepsilon_{m}$, respectively. 

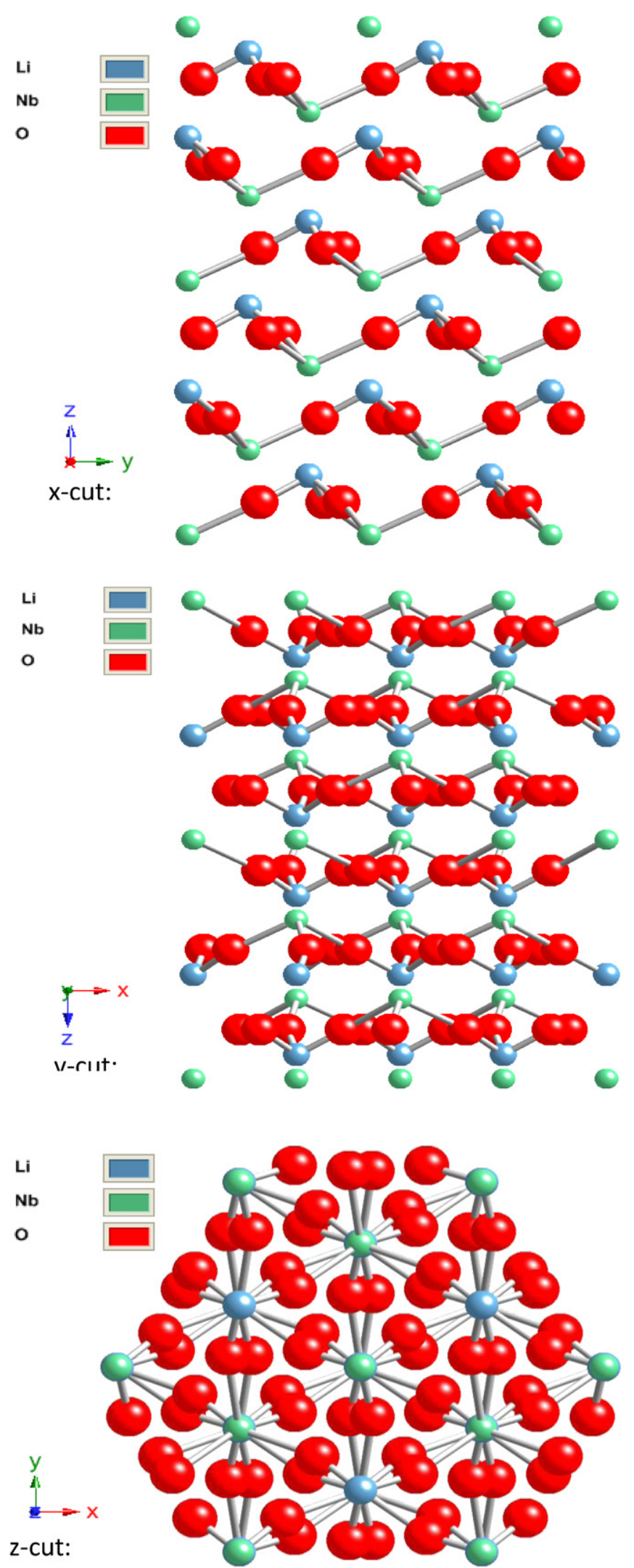

Figure 3. Crystallographic structure of $\mathrm{LiNbO}_{3}$ showing the projection on the $Y Z$ plane ( $X$-cut), on the $X Z$ plane ( $Y$-cut) and on the $X Y$ plane ( $Z$-cut). adopt a simpler zero-order approximation and assume that the displacement field $\boldsymbol{u}(r, \theta)$ at the halo and core, and hence the strain tensors present axial symmetry around the corresponding axis (as for the isotropic case); however, one should use appropriate elastic coefficients (e.g. Poisson ratios) for each particular track orientation. According to this conjecture the angular profiles for the displacement field neglects all highorder harmonics except the zero-order one. On the other hand, the effect of crystal anisotropy on the stress tensors will be taken into account through the anisotropic elastic stiffness and compliance coefficients. We will show next the usefulness of such a simple approach that allows one to obtain practical quantitative predictions for a number of experimental scenarios.

\subsection{Strain tensors}

In accordance with the above main assumption, expression (2) (referred to the strain tensor for tracks along the $X, Y$ and $Z$ crystal axes) remains valid for the anisotropic case. However, the physical factor, $g=1 / 2(1+v) /(1-v)$, in that equation should now include the appropriate Poisson coefficient for each particular track orientation. Then, using a comparison between the isotropic and anisotropic formulae (10) for the components of the strain tensor and the values for the compliance coefficients in table 1, we obtain the following anisotropic Poisson ratios and physical factors:

$\begin{array}{ll}v_{X Y}=v_{Y X}=-S_{12} / S_{11}=0.175, & g_{X Y}=g_{Y X}=0.712 \\ v_{X Z}=v_{Y Z}=-S_{13} / S_{11}=0.254, & g_{X Z}=g_{Y Z}=0.841 \\ v_{Z X}=v_{Z Y}=-S_{13} / S_{33}=0.293, & g_{Z X}=g_{Z Y}=0.914 .\end{array}$

Hence the symmetry of the strain tensor $\varepsilon$ around the track axis remains isotropic for track $Z$ but develops a slight anisotropy for tracks $X$ and $Y$, due to the different $g$ factors, i.e. $g_{x z} \neq g_{z x}$ and $g_{y z} \neq g_{z y}$. Anyhow, one can take an average factor $g_{\text {av }}=(0.841+0.914) / 2=0.878$ and consider the strain field as very approximately isotropic also for tracks along the $X$ - and $Y$-axes. We summarize here the final expressions for the strain tensors.

Track along the Z-axis:

$$
\varepsilon(r, \theta)=0.712 \cdot \varepsilon_{0} \frac{R^{2}}{r^{2}}\left(\begin{array}{ccc}
-\cos 2 \theta & -\sin 2 \theta & 0 \\
-\sin 2 \theta & \cos 2 \theta & 0 \\
0 & 0 & 0
\end{array}\right) .
$$

Track along the $X$-axis:

$$
\varepsilon(r, \theta)=0.878 \cdot \varepsilon_{0} \frac{R^{2}}{r^{2}}\left(\begin{array}{ccc}
0 & 0 & 0 \\
0 & -\cos 2 \theta & -\sin 2 \theta \\
0 & -\sin 2 \theta & \cos 2 \theta
\end{array}\right) .
$$

Track along the Y-axis:

$$
\varepsilon(r, \theta)=0.878 \cdot \varepsilon_{0} \frac{R^{2}}{r^{2}}\left(\begin{array}{ccc}
-\cos 2 \theta & 0 & -\sin 2 \theta \\
0 & 0 & 0 \\
-\sin 2 \theta & 0 & \cos 2 \theta
\end{array}\right) .
$$

Therefore, as a main conclusion, the strains are scaled by a factor dependent on the Poisson ratio applicable to each particular track orientation. 


\subsection{Stress tensors}

For the anisotropic situation considered here for $\mathrm{LiNbO}_{3}$, the track direction is relevant for the calculation of the stress-tensor components. One readily obtains these components making use of (11) and the appropriate equations (12)-(14) for a given track direction.

\section{Track along the Z-axis:}

$\boldsymbol{\sigma}(r, \theta)=0.712 \cdot \varepsilon_{0} \frac{R^{2}}{r^{2}}$

$\times\left(\begin{array}{ccc}\left(C_{12}-C_{11}\right) \cos 2 \theta & -C_{66} \sin 2 \theta & -2 C_{41} \sin 2 \theta \\ -C_{66} \sin 2 \theta & \left(C_{11}-C_{12}\right) \cos 2 \theta & -2 C_{41} \cos 2 \theta \\ -2 C_{41} \sin 2 \theta & -2 C_{41} \cos 2 \theta & 0\end{array}\right)$.

Neglecting the out of plane $(X Y)$ components of the tensor that mix with the $Z$ components (i.e. the comparatively small $C_{41}$ coefficient), the stress tensor becomes

$$
\begin{aligned}
\boldsymbol{\sigma}(r, \theta) & =0.712 \cdot \varepsilon_{0} \frac{R^{2}}{r^{2}} \\
\times & \times\left(\begin{array}{ccc}
\left(C_{12}-C_{11}\right) \cos 2 \theta & -C_{66} \sin 2 \theta & 0 \\
-C_{66} \sin 2 \theta & \left(C_{11}-C_{12}\right) \cos 2 \theta & 0 \\
0 & 0 & 0
\end{array}\right)
\end{aligned}
$$

which has the same structure as for the isotropic case (3). Normal and shear stresses over a plane whose normal vector forms an angle $\alpha$ with the $X$-axis (see figure 1) are given by

$$
\begin{aligned}
\sigma_{N}(\alpha)= & 0.712 \cdot \varepsilon_{0} \frac{R^{2}}{r^{2}} \\
& \times\left[\left(C_{12}-C_{11}\right) \cos 2 \theta \cos 2 \alpha-C_{66} \sin 2 \theta \sin 2 \alpha\right] \\
= & 0.712 \cdot \varepsilon_{0} \frac{R^{2}}{r^{2}}\left[\left(C_{12}-C_{11}\right) \cos 2 \beta\right] \\
\sigma_{S}(\alpha)= & 0.712 \cdot \varepsilon_{0} \frac{R^{2}}{r^{2}} \\
& \times\left[-\left(C_{12}-C_{11}\right) \cos 2 \theta \sin 2 \alpha-C_{66} \sin 2 \theta \cos 2 \alpha\right] \\
= & 0.712 \cdot \varepsilon_{0} \frac{R^{2}}{r^{2}}\left[\left(C_{12}-C_{11}\right) \sin 2 \beta\right]
\end{aligned}
$$

where $\beta=\alpha-\theta$, as defined in section 2 above.

For $\theta=0$ ( $X$-axis) the angular profiles as a function of $\alpha$, depicted in figure 4 , follow the same patterns as for the isotropic case (see figure 2). In particular, one should note that the absolute value of the maximum normal and shear stresses are independent of $\theta$ as for the isotropic case, i.e. there are no privileged planes in the structure.

Track along the $X$-axis:

$$
\begin{aligned}
& \boldsymbol{\sigma}(r, \theta)=0.878 \cdot \varepsilon_{0} \frac{R^{2}}{r^{2}} \\
& \left(\begin{array}{ccc}
\left(C_{13}-C_{12}\right) \cos 2 \theta & 0 & 0 \\
-C_{14} \sin 2 \theta & & \\
0 & \left(C_{13}-C_{11}\right) \cos 2 \theta & C_{41} \cos 2 \theta \\
& +C_{14} \sin 2 \theta & -C_{44} \sin 2 \theta \\
0 & C_{41} \cos 2 \theta & \left(C_{33}-C_{31}\right) \\
& -C_{44} \sin 2 \theta & \times \cos 2 \theta
\end{array}\right) .
\end{aligned}
$$
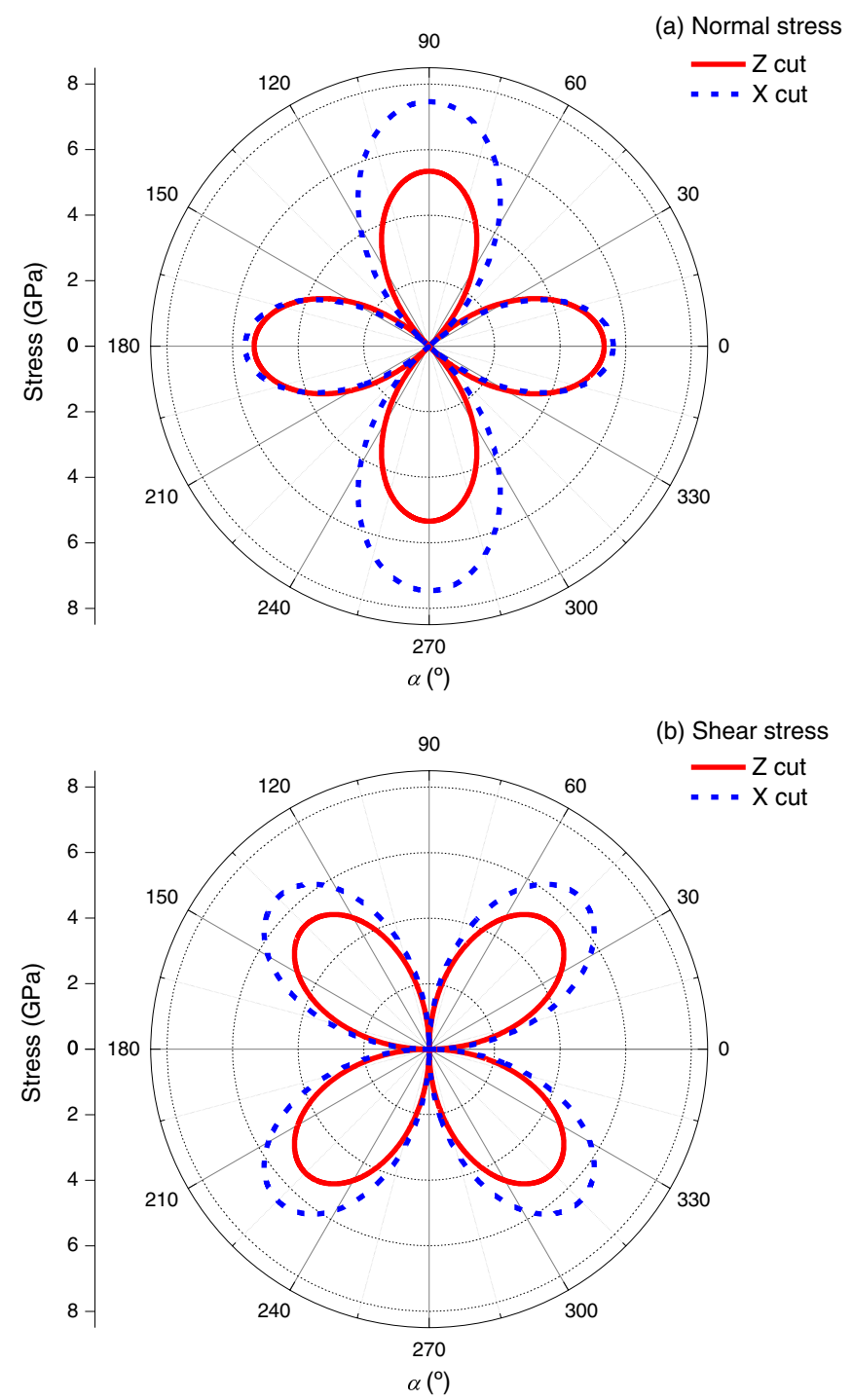

Figure 4. (a) Absolute value of the normal stress produced by a track along $Z$ (and $X$ )-axis at a point $r=R$ and $\theta=0$ over a probe plane whose normal vector forms an angle $\alpha$ with the $X$-axis

( $Y$-axis). (b) Absolute values of the shear stress produced by a track along $Z$ (and $X$ )-axis at a point $r=R$ and $\theta=0$ over a probe plane whose normal vector forms an angle $\alpha$ with the $X$-axis ( $Y$-axis).

It is convenient here to neglect the small stress component $\sigma_{x x}$ and restrict our analysis to the $Y Z$ plane. Now, the offdiagonal terms mixing the $Y$ and $Z$ components of the stress become more relevant. Even neglecting such off-diagonal terms, the equivalence of $Y$ - and $Z$-axes in the perpendicular plane is broken since the diagonal terms do not coincide. For simplicity, we neglect the coefficients $C_{14}$ and $C_{41}$ and the $\sigma_{x x}$ element, not introducing much error due to their small values. The resulting stress matrix can thus be written as

$$
\begin{aligned}
\boldsymbol{\sigma}(r, \theta) & =0.878 \cdot \varepsilon_{0} \frac{R^{2}}{r^{2}} \\
& \times\left(\begin{array}{ccc}
0 & 0 & 0 \\
0 & \left(C_{13}-C_{11}\right) \cos 2 \theta & -C_{44} \sin 2 \theta \\
0 & -C_{44} \sin 2 \theta & \left(C_{33}-C_{31}\right) \cos 2 \theta
\end{array}\right) .
\end{aligned}
$$

One then gets for the normal and shear stresses as a function 


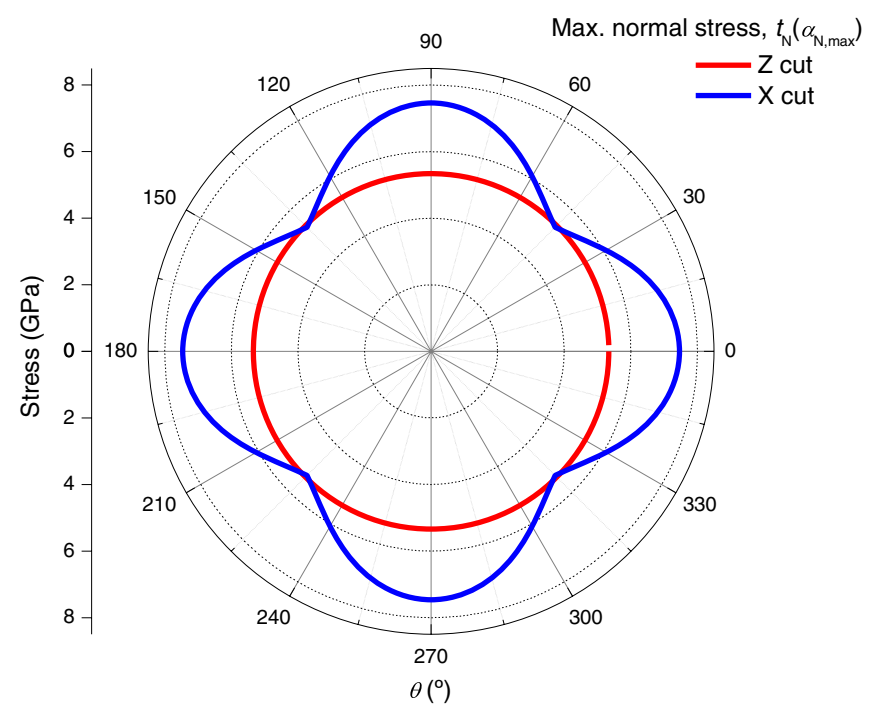

Figure 5. Maximum normal stress produced by a track along $Z$ (and $X)$-axis at points $(r=R, \theta)$.

of $\theta$ and $\alpha$

$$
\begin{aligned}
\sigma_{\mathrm{N}}= & 0.878 \cdot \varepsilon_{0} \frac{R^{2}}{r^{2}}\left[-C_{44} \sin 2 \theta \sin 2 \alpha\right. \\
& \left.+\cos 2 \theta\left(C_{13} \cos 2 \alpha+C_{33} \sin ^{2} \alpha-C_{11} \cos ^{2} \alpha\right)\right] \\
\sigma_{\mathrm{S}}= & 0.878 \cdot \varepsilon_{0} \frac{R^{2}}{r^{2}}\left[-C_{44} \sin 2 \theta \cos 2 \alpha\right. \\
& \left.+\frac{1}{2}\left(C_{33}+C_{11}-2 C_{13}\right) \cos 2 \theta \sin 2 \alpha\right]
\end{aligned}
$$

i.e., the $\alpha$-angular profiles depend on $\theta$ and so they do not present axial symmetry as for the track along the $Z$-axis (or the isotropic case). As an example the profiles for $\theta=0$ are

$\sigma_{\mathrm{N}}=0.878 \cdot \varepsilon_{0} \frac{R^{2}}{r^{2}}\left(C_{13} \cos 2 \alpha+C_{33} \sin ^{2} \alpha-C_{11} \cos ^{2} \alpha\right)$,

$\sigma_{\mathrm{S}}=0.878 \cdot \varepsilon_{0} \frac{R^{2}}{r^{2}}\left[\left(C_{33}+C_{11}-2 C_{13}\right) \frac{\sin 2 \alpha}{2}\right]$.

The polar plots (as a function of $\alpha$ ) for the normal stresses at $\theta=0$ for tracks along the $X$ - and $Z$-axes are comparatively illustrated in figure 4(a). The corresponding plots for the shear stresses are displayed in figure $4(b)$. Note that for the $Z$ cut the angular profiles are similar to those for the isotropic case (figure 2) except for the scaling factor in magnitude. The anisotropy in the normal stress obtained for tracks along the $X$-axis is remarkable. Irradiations along the $X$-axis provide higher maximum normal and shear stress values than along the $Z$-axis.

For practical purposes the most interesting information refers to the maximum values for both stresses, $\sigma_{\mathrm{N}}$ (figure 5) and $\sigma_{\mathrm{S}}$ (figure 6), which take place on planes characterized by their angles $\alpha_{\mathrm{N} \text {,max }}$ and $\alpha_{\mathrm{S} \text {,max }}$, respectively. The orientation and magnitude for both the maximum normal and shear stresses are shown as a function of $\theta$ formed by the observation direction with the $X(Y)$ crystal axis for $Z$-cut ( $X$-cut). In the case of shear stress the orientation of the maximum stress with respect to the $X(Y)$-axis is also given for $Z$-cut ( $X$-cut).

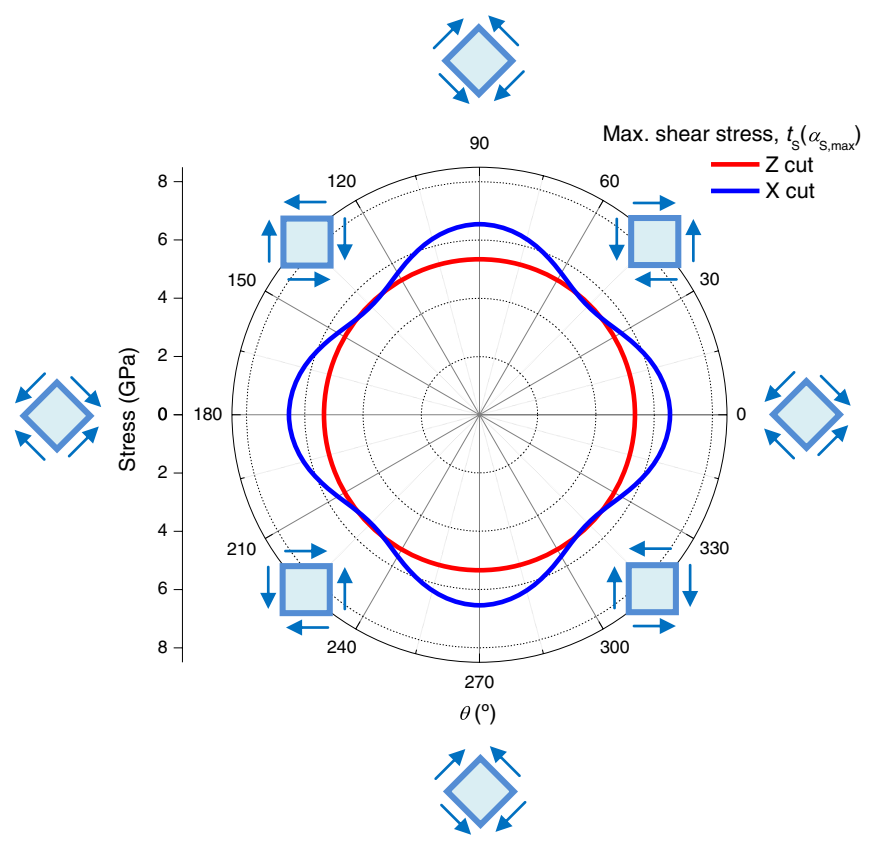

Figure 6. Maximum shear stress produced by a track along $Z$ (and $X)$-axis at points $(r=R, \theta)$. The squares represent infinitesimal elements over which maximum stress acts. The arrows represent the shear stress. Note that the maximum shear stress appears on planes forming $\pm 45^{\circ}$ with the crystallographic axes.

One sees that the axial isotropy is lost in contrast to the situation for tracks along the $Z$-axis. It turns out that the normal stress reaches the highest values at points on the $Y$ - or $Z$-axis. In the case of shear stress the highest values always occur along directions forming $\pm 45^{\circ}$ with those axes, as schematically illustrated in figure 6 . In other words, the anisotropy has destroyed the equivalence among all crystal planes (regardless of $\alpha$ ) and has selected a set of 'weaker' shear planes. This prediction is reasonably consistent with the appearance of cracks at $\pm 45^{\circ}$ upon irradiation of $X$-cut samples, as will be discussed in section 4.4 (see figure 9).

Track along the Y-axis:

$$
\begin{gathered}
\sigma(r, \theta)=0.878 \cdot \varepsilon_{0} \frac{R^{2}}{r^{2}} \\
\times\left(\begin{array}{cc}
\left(C_{13}-C_{11}\right) \cos 2 \theta & -C_{14} \sin 2 \theta \\
-C_{14} \sin 2 \theta & \left(C_{13}-C_{12}\right) \cos 2 \theta \\
-C_{44} \sin 2 \theta & -C_{41} \cos 2 \theta \\
-C_{44} \sin 2 \theta \\
-C_{41} \cos 2 \theta \\
\left(C_{33}-C_{31}\right) \cos 2 \theta
\end{array}\right) .
\end{gathered}
$$

The situation is very similar to that for the track along $X$. Again, even neglecting the off-diagonal terms, the axial symmetry (or equivalence of $X$ - and $Z$-axes) is destroyed.

\section{Consequences of the anisotropic strain/stress fields around amorphous tracks}

In principle, the elastic halo affects many physical and chemical properties of the crystal such as microscopy observations (TEM), x-ray diffraction, optical parameters 


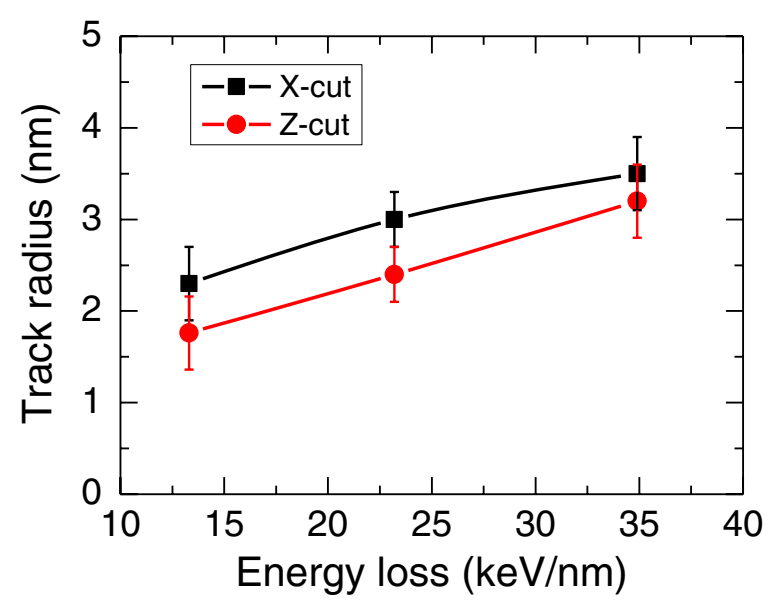

Figure 7. Comparative plot showing the track radii measured by $\mathrm{RBS} / \mathrm{C}$ for $X$ - and $Z$-cut $\mathrm{LiNbO}_{3}$ plates for irradiation with ions of different stopping powers.

(refractive index), channelling behaviour of incoming light ions or chemical reactivity. Unfortunately, a systematic study of such effects is not yet available, although some partial information is scattered through the literature.

\subsection{Microscopy observations}

First, TEM images could provide clear details about the crystal structure in the track and about the lattice distortions at the surrounding halo. In fact, clear evidence of heavy strains around the track core was reported [46, 47] for GeS irradiated with $\mathrm{U}$ ions at $11.4 \mathrm{MeV} \mathrm{amu}^{-1}$. For $\mathrm{LiNbO}_{3}$, significant structural changes were observed in the diffraction patterns obtained with HRTEM $[28,61]$. In fact, the track radii measured by TEM are smaller (about a factor 2) than those determined by RBS/C. The latter technique does not distinguish individual tracks but measures the total disordered area corresponding to a given (small) fluence $\left(\sim 10^{10} \mathrm{~cm}^{-2}\right)$. This area should include not only the amorphous track core, as in TEM, but a substantial fraction of the surrounding disordered halo. Our theoretical analysis predicts higher strain/stress fields for tracks oriented along the $X$ - or $Y$-axis in comparison with those associated with tracks along $Z$ due to differences in the effective Poisson coefficients. The available information is still very scarce, hence we have performed RBS/C experiments at stopping powers well above the threshold, irradiating with $\mathrm{Br}(45 \mathrm{MeV})$ in our $5 \mathrm{MV}$ accelerator at CMAM and with Xe $(1.45 \mathrm{GeV}), \mathrm{Kr}(800 \mathrm{MeV})$ and $\mathrm{Au}(2.19 \mathrm{GeV})$ at GANIL. For these irradiations one expects a relevant contribution of the elastic halo. The results corresponding to irradiations on the $X$ and $Z$ crystal cuts at the same low fluences are shown in figure 7 . They confirm that the disorder area associated with a given track is, indeed, larger for irradiations along the $X$-axis than along the $Z$-axis, although the difference is not very significant. Anyhow, one cannot rule out the contribution of other effects to the observed differences in track morphology, e.g. anisotropy of the damage mechanism and/or size of the defective halo. Systematic experiments using TEM and complementary AFM methods should be pursued.

\subsection{Surface swelling}

An effect related to track formation is the surface swelling effect observed at the impact spot on the irradiated face of the crystal [48-50]. It is revealed as a nanometric hillcock around the emergence point of the track at the surface or as a raised mesa or plateau when a finite region is homogeneously irradiated. These protrusions are a consequence of the strains generated at the free sample surface by the amorphous track [40] and should be governed by the Poisson coefficients, relating the compressive stresses at the perpendicular plane to the induced strains along the track direction. Therefore, swelling measurements appear very suitable to investigate the elastic distortions associated with amorphous tracks. The problem of swelling caused by tracks in a semi-infinite solid has been dealt with by Colin et al [40] using a Green's method approach under an isotropic approximation. It appears [43] that the radial extension of the hillcock (elastic) profile at the surface amounts to around twice the radius of the amorphous core. However, their results would require a more general theoretical framework to deal with anisotropic crystals such as $\mathrm{LiNbO}_{3}$. Here, we propose a simple quasi-isotropic analysis based on the formalism of the effective Poisson coefficients. One shortcoming of this analysis is that it neglects the relatively small stresses parallel to the track axis. Anyway, the analysis is expected to provide a reasonable approximation, especially, when comparing tracks oriented along different crystal axes. For cylindrical tracks along a principal crystal axis, perpendicular to the free surface and emerging at it, the magnitude of swelling measured by the normal displacement $u_{\mathrm{N}}$ of the surface at the track axis $(r=0)$ is [40]

$$
u_{\mathrm{N}}=2(1+v) \varepsilon_{0}\left\{h+a-\left(a^{2}+h^{2}\right)^{1 / 2}\right\}
$$

where $v$ is the effective Poisson ratio for the experimental configuration, $\varepsilon_{0}$ the radial strain inside the track, $a$ the radius of the track and $h$ its length. Therefore, one would expect swelling values around $30 \%$ higher for $X$-tracks in comparison with $Z$-tracks. As far as we know, sufficient reliable data are not yet available. Swelling ratios, $u_{\mathrm{N}}(X$ cut $) / u_{\mathrm{N}}(Z$ cut $)=$ $1-2$, were, indeed, observed in old experiments [62] on $\mathrm{LiNbO}_{3}$ crystals irradiated with nitrogen at $150 \mathrm{keV}$, i.e. with a strong collisional contribution. In order to ensure that we are in the electronic regime and to enhance the swelling magnitude, we have performed experiments on samples irradiated at a moderate fluence in our $5 \mathrm{MV}$ accelerator at CMAM with $\mathrm{F}$ at $20 \mathrm{MeV}$ and $\mathrm{Br}$ at $45 \mathrm{MeV}$. In these experiments the irradiated area has a radius, $a \gg h$, of around $1 \mathrm{~mm}$. Then, formula (26) turns into

$$
u_{\mathrm{N}} \propto(1+v) \varepsilon_{0} h .
$$

Our results, shown in figure 8 , reveal that the amount of surface swelling at the $X$ face is about a factor 1.3 larger than at the $Z$ face, as expected from the theoretical analyses presented in this paper. Moreover, the absolute values of the swelling magnitude have the correct order of magnitude $(\sim 100 \mathrm{~nm})$. Single track swelling experiments comparing hillcock profiles for single tracks along different orientations will be quite relevant to confirm these predictions. 


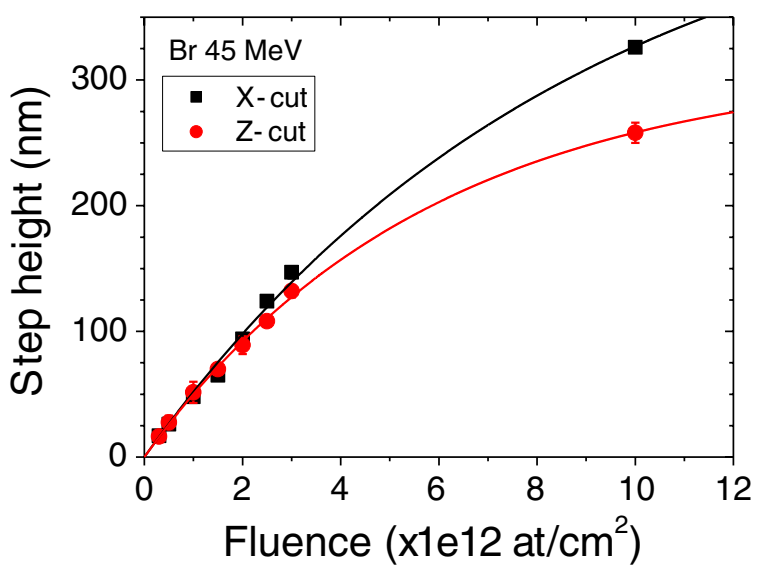

Figure 8. Magnitude of the swelling heights measured by profilometry for $X$ - and $Z$-cut $\mathrm{LiNbO}_{3}$ plates irradiated with $\mathrm{Br}$ at $45 \mathrm{MeV}$.

\subsection{X-ray diffraction}

The strain fields around the core track induce changes in the lattice parameters of the surrounding crystalline material (halo) that could be measured by high-resolution x-ray diffraction. The observable consequences will be a shift and a broadening of the Bragg peaks. The expected shift can be roughly estimated in a simple model of a cubic lattice of parameter $l$. For a single track, the decrease in the lattice parameter $l$, averaged over the extension of the halo, yields

$$
\begin{aligned}
\frac{\langle\Delta l\rangle}{l} & =\frac{1}{\pi\left(R_{\mathrm{H}}^{2}-R_{\mathrm{C}}^{2}\right)} \int_{R_{\mathrm{C}}}^{R_{\mathrm{H}}} \frac{\Delta l(r)}{l} \cdot 2 \pi r \mathrm{~d} r \\
& =\frac{R_{\mathrm{C}}^{2}}{R_{\mathrm{H}}^{2}-R_{\mathrm{C}}^{2}} \varepsilon_{0} \frac{1+v}{1-v} \ln \left(\frac{R_{\mathrm{H}}}{R_{\mathrm{C}}}\right)
\end{aligned}
$$

since $\Delta l / l=\varepsilon_{r r} . R_{\mathrm{H}}$ and $R_{\mathrm{C}}$ are, respectively, the radii of the halo and core of the tracks. The average relative change in lattice parameter is not dramatically dependent on the ratio between the halo and core radii. For the reasonable choice $R_{\mathrm{H}}=1.5 R_{\mathrm{C}},\langle\Delta l\rangle / l \cong 2.5 \times 10^{-2}$, whereas it would decrease to $2 \times 10^{-3}$ for the extreme case of $R_{\mathrm{H}}=10 R_{\mathrm{C}}$. Therefore, the values to be found in experiment would depend on the coverage of the halos. For high enough fluences, so that the average distance between impacts is of the order of the halo radius, one would reach a value between the two limits mentioned above. We may, here, quote data obtained [63] by researchers at the University of Padova for $\mathrm{LiNbO}_{3}$ samples irradiated at our accelerator (CMAM) with F ions at $20 \mathrm{MeV}$ along the $Z$-axis to a fluence of $10^{14} \mathrm{~cm}^{-2}$. They obtained values ranging from 1 to $5 \times 10^{-3}$. MicroRaman experiments performed $[3,6]$ on different swift ion-beam irradiated $\mathrm{LiNbO}_{3}$ samples also show a broadening and shift of the crystalline peaks presumably due to the strains existing in the crystalline regions in the vicinity of the amorphous regions. Experiments on other oxides, such as zirconia [51], have reported strains of $\sim 2 \times 10^{-3}$ for $\mathrm{Pb}$-irradiated samples at fluences of $\sim 10^{13} \mathrm{~cm}^{-2}$.

\subsection{Mechanical effects}

The strain/stress fields in the halo may induce mechanical effects, e.g. generation of dislocations, as well as initiate or propagate fracture cracks. Glide deformation [62] has been observed on congruent $\mathrm{LiNbO}_{3}$ only for temperatures around or above $1000^{\circ} \mathrm{C}\left(0.8 T_{\mathrm{m}}, T_{\mathrm{m}}\right.$ being the melting temperature $)$. It occurs along different slip planes, basal, prismatic and pyramidal, depending on the compression axis. The measured yield stress measured at such high temperatures is in the 13-20 MPa range [64] significantly lower than the values obtained from our calculations at the track boundary, where temperature reaches the highest values (near the melting point) for a very short time. At lower temperatures plastic deformation could not be achieved without failure [64]. In particular, these mechanical effects should show a clear dependence on the sample cut that has been exposed to irradiation, either $X, Y$ or $Z$. In fact, the main consequence of our analysis of the anisotropy is that the stresses around a track are higher for $X$ - or $Y$-cuts in comparison with $Z$-cuts. This could explain the clear fracture patterns observed on $X$-oriented tracks in comparison with $Z$-oriented tracks for swift-ion irradiations at equal fluences. This effect is illustrated with photographs in figure 9 showing the $X$ - and $Z$-faces irradiated with Xe ions at fluences of 2 and $8 \times 10^{11} \mathrm{~cm}^{-2}$. The crack patterns are oriented at around $\pm 45^{\circ}$ with respect to the axes, in agreement with the orientation of the highest values obtained for the maximum shear stresses (figure 6).

\subsection{Chemical effects}

The stress/strain field profiles could also influence the surface morphology patterns of tracks after suitable chemical etching [28], which results in the formation of nanopores at the sample surface. It has been observed that the initial pore shapes, essentially corresponding to the excavation of the amorphous core, are axial (circular). However, during subsequent pore growth other anisotropic shapes develop and may adopt a markedly elongated shape with the long axis at around $40^{\circ}$ from the $Z$-axis [28]. This value is close to the predictions of our anisotropic analysis for maximum shear stresses, suggesting that those directions present a weaker resistance to chemical etching. The marked pore anisotropy is particularly observable for samples that have been irradiated with heavy ions of very high energy for which the electronic stopping power leads to thick amorphous tracks and the size of the elastic halo may be large. This is illustrated by the morphology of the etched tracks in figure 10, corresponding to samples irradiated at GANIL with $\mathrm{Kr} 800 \mathrm{MeV}$ and $\mathrm{Pb} 2300 \mathrm{MeV}$, using $\mathrm{HF}$ (40\%) as the etching agent for $10 \mathrm{~min}$ at RT.

\section{Summary and outlook}

The stress/strain profiles around a single amorphous track in a $\mathrm{LiNbO}_{3}$ crystal irradiated with swift heavy ions at normal incidence along the principal crystal axes are evaluated. First, the situation is reviewed within an isotropic model, in order to assess the concept of elastic halo and its relative relevance in comparison with the defective (point defect) halo. This is a key problem in the analysis of experiments since the two contributions are entangled and are difficult to distinguish. In a second part, the effect of crystal anisotropy is taken into 


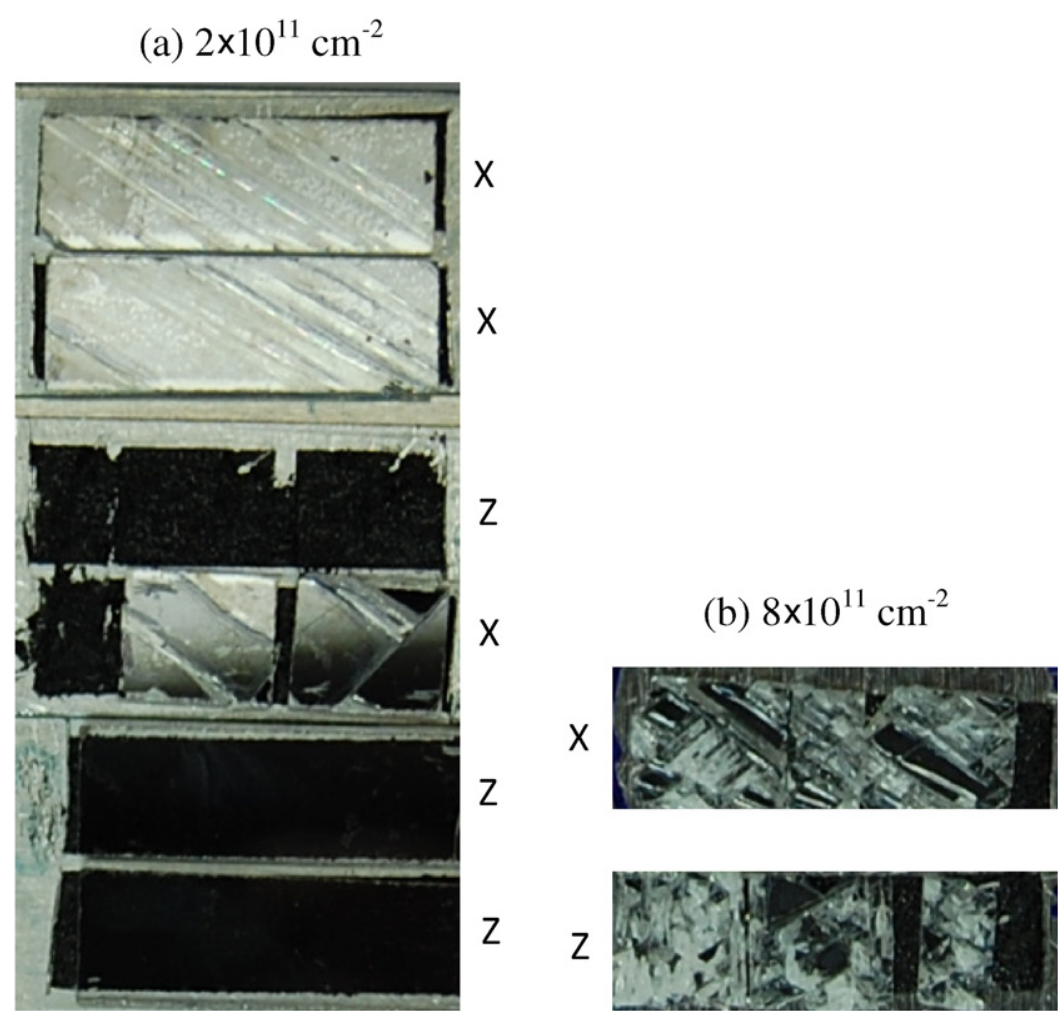

Figure 9. Photographs of cracks developing at around $\pm 45^{\circ}$ on $X$-cut samples upon irradiation with Xe ions of energy $11.1 \mathrm{MeV} \mathrm{amu}^{-1}$ at fluences of $2 \times 10^{11} \mathrm{~cm}^{-2}(a)$ and $8 \times 10^{11} \mathrm{~cm}^{-2}(\mathrm{~b})$. For comparison $Z$-cut samples are included. In the latter case surface damage is observed at high fluence $\left(8 \times 10^{11} \mathrm{~cm}^{-2}\right)$ but follows no clear pattern unlike the $X$-cut samples. All the samples were glued on metallic holders by means of a graphite tape.
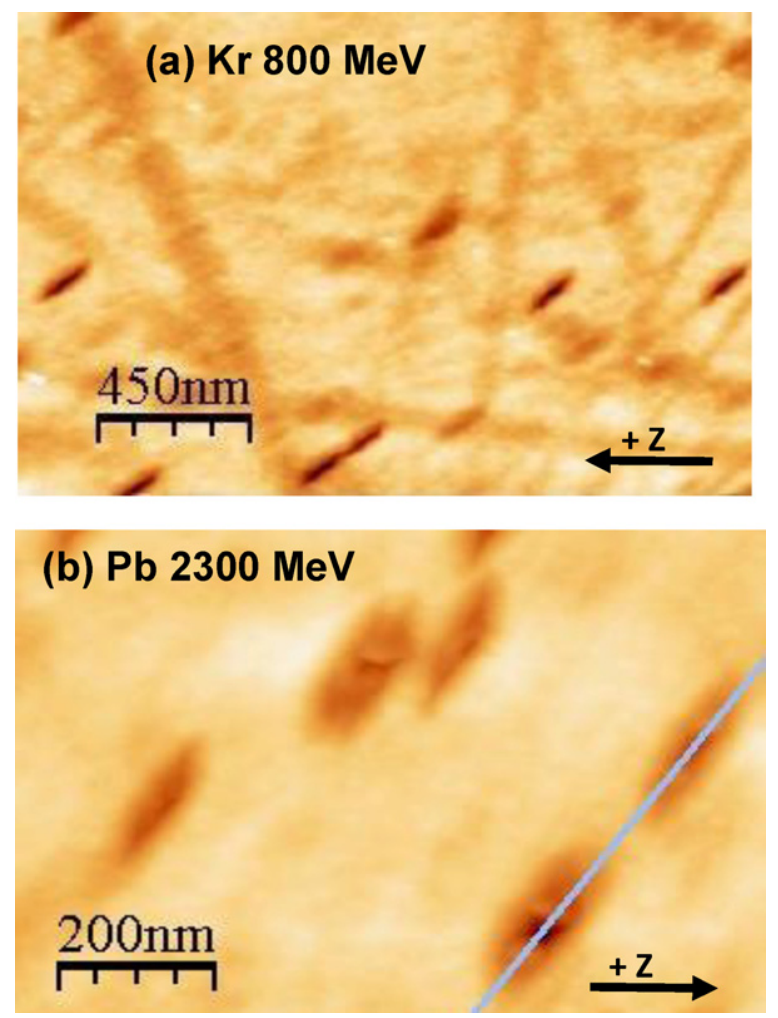

Figure 10. AFM images of elongated pores generated by irradiation with $\mathrm{Kr}$ at $800 \mathrm{MeV}$ and $\mathrm{Pb}$ at $2.3 \mathrm{GeV}$, followed by chemical etching in $40 \% \mathrm{HF}$. account through a practical approximate model. It assumes an axial displacement (and strain) field that depends on the orientation of the track through an effective Poisson ratio. The contributions to the non-axial terms in the stress radial profiles are then calculated. The model accounts for isotropic stress profiles around a track along the $Z$-axis and predicts a higher stress field around tracks along the $X$ - and $Y$-axes. In addition, in the latter cases, the maximum normal and shear stresses do not present radial isotropy. The results provide a basis to understand certain relevant experimental effects on the role of track orientation (such as the size of the disordered area, surface swelling, fracture patterns or chemical etching of amorphous tracks). Moreover, it offers interesting predictions on the effect of irradiation tracks on lattice parameters and $\mathrm{x}$-ray diffraction patterns.

\section{Acknowledgments}

The authors express their deep appreciation to M Toulemonde, E Balanzat and other members of the GANIL laboratory for performing some of the irradiations analysed in this work.

\section{References}

[1] Bentini G G, Bianconi M, Chiarini M, Correra L, Sada C, Mazzoldi P, Argiolas N, Bazzan M and Guzzi R 2002 J. Appl. Phys. 926477 
[2] Bentini G G, Bianconi M, Correra L, Chiarini M, Mazzoldi P, Sada C, Argiolas N, Bazzan M and Guzzi R 2004 J. Appl. Phys. 96242

[3] Olivares J, García G, Agulló-López F, Agulló-Rueda F, Kling A and Soares J C 2005 Appl. Phys. A 811465

[4] Agulló-López F, García G and Olivares J 2005 J. Appl. Phys. 9793514

[5] García-Navarro A, Olivares J, García G, Agulló-López F, García-Blanco S, Merchant C and Stewart Aitchison J 2006 Nucl. Instrum. Methods Phys. Res. B 249177

[6] Olivares J, García-Navarro A, García G, Agulló-López F, Agulló-Rueda F, García-Cabañes A and Carrascosa M 2007 J. Appl. Phys. 10133512

[7] Chen F 2009 J. Appl. Phys. 106081101

[8] Rivera A, Olivares J, García G and Agulló-López F 2008 Nucl. Instrum. Methods Phys. Res. B 2662859

[9] Rivera A, Olivares J, Crespillo M L, García G, Bianconi M and Agulló-López F 2009 Nucl. Instrum. Methods Phys. Res. B 2671460

[10] Rivera A, Olivares J, García G, Cabrera J M, Agulló-Rueda F and Agulló-López F 2009 Phys. Status Solidi a 2061109

[11] Jensen J, Skupinski M, Hjort K and Sanz R 2000 Nucl. Instrum. Methods Phys. Res. B 164/165 377

[12] Komarov F, Gaiduk P and Kamarou A 2001 Vacuum 63657

[13] Wesch W, Kamarou A and Wendler E 2004 Nucl. Instrum. Methods Phys. Res. B 225111

[14] Kamarou A, Wesch W, Wendler E, Unsdisz A and Rettenmayr M 2006 Phys. Rev. B 73184107

[15] Bianconi M, Argiolas N, Bazzan M, Bentini G G, Chiarini M, Cerutti A, Mazzoldi P, Pennestri G and Sada C 2005 Appl. Phys. Lett. 87072901

[16] Canut, Ramos S M M, Brenier R, Thevenard P, Loubet J L and Toulemonde M 1996 Nucl. Instrum. Methods Phys. Res. B 107194

[17] Meftah A, Constantini J M, Khalafoui N, Boudjadar S, Stoquert J P, Studer F and Toulemonde M 2005 Nucl. Instrum. Methods Phys. Res. B 237563

[18] Vetter J, Scholz R and Angert N 1994 Nucl. Instrum. Methods Phys. Res. B 91129

[19] Thibaud F, Cousty J, Balanzat E and Bouffard S 1991 Phys. Rev. Lett. 671582

[20] Ackermann J, Angert N, Neumann R, Trautmann C, Dischner M, Hagen T and Sedlacek M 1996 Nucl. Instrum. Methods Phys. Res. B 107181

[21] Abu Saleh S and Eyal Y 2004 Appl. Phys. Lett. 852529

[22] Albrecht D, Armbruster P, Spohr R, Roth M, Schaupert K and Stuhrmann H 1985 Appl. Phys. A 3737

[23] Meftah A, Brisard F, Constantini J M, Hage-Ali M, Stoquert J P and Toulemonde M 1993 Phys. Rev. B 48920

[24] Garcia-Navarro A, Agulló-López F, Bianconi M, Olivares J and García G 2007 J. Appl. Phys. 101083506

[25] Toulemonde M, Fuchs G, Nguyen N, Studer F and Groult D 1987 Phys. Rev. B 356560

[26] Assmann W, Dobler M, Avasth D K, Kruijer S, Mieskes H D and Nolte H 1998 Nucl. Instrum. Methods Phys. Res. B 146271

[27] Olivares J, García-Navarro A, García G, Méndez A and Agulló-López F 2006 Appl. Phys. Lett. 89071923

[28] Crespillo M L, Otto M, Muñoz-Martin A, Olivares J, Agulló-López F, Seibt M, Toulemonde M and Trautmann C 2009 Nucl. Instrum. Methods Phys. Res. B 2671035

[29] Dong N, Chen F, Jaque D, Benayas A, Qiu F and Narusawa T 2011 J. Phys. D: Appl. Phys. 44105103

[30] Zollondz J H and Weidinger A 2004 Nucl. Instrum. Methods Phys. Res. B 225178

[31] Toulemonde M, Trautmann C, Balanzat E, Hjort K and Weidinger A 2004 Nucl. Instrum. Methods Phys. Res. B 2161
[32] Nesprías F, Venturino M, Debray M E, Davidson J, Davidson M, Kreiner A J, Minsky D, Fischer M and Lamagna A 2009 Nucl. Instrum. Methods Phys. Res. B 26769

[33] Toulemonde M, Assman W, Dufour C, Meftah A, Studer F and Trautmann C 1996 Ion Beam Science: Solved and Unsolved Problems ed P Sigmund (Copenhagen: The Royal Danish Academy of Sciences and Letters) pp 263-92

[34] Szenes G 1995 Phys. Rev. B 518026

[35] Itoh N 1998 Nucl. Instrum. Methods Phys. Res. B 135175

[36] Agulló-López F, Méndez A, García G, Olivares J and Cabrera J M 2006 Phys. Rev. B 74174109

[37] Rivera A, Méndez A, García G, Olivares J, Cabrera J M and Agulló-López F 2008 J. Lumin. 128703

[38] Eshelby J D 1957 Proc. R. Soc. A 241376

[39] Eshelby J D 1959 Proc. R. Soc. A 252561

[40] Colin J, Lesueur D and Grilhé J 2001 Phil. Mag. A 81857

[41] Mura T 1987 Micromechanics of Defects in Solids 2nd edn (Dordrecht: Martinus Nijhoff)

[42] Ryazanov A I, Volkov A E and Klaumünzer S 1995 Phys. Rev. B 5112107

[43] Trinkaus H 1998 Nucl. Instrum. Methods Phys. Res. B 146204

[44] Bursill L A and Braunhausen G 1990 Phil. Mag. A 62395

[45] Canut B, Brenier R, Meftah A, Moretti P, Ould Salem S, Pitaval M, Ramos S M M, Thevenard P and Toulemonde M 1995 Radiat. Eff. Defects Solids 136307

[46] Vetter J, Scholz R, Dobrev D and Nistor L 1998 Nucl. Instrum. Methods. B 141747

[47] Trautmann C 1999 Bull. Mater. Sci. 22679

[48] Adouard A, Mamy R, Toulemonde M, Szenes G and Thomé L 1997 Europhys. Lett. 40527

[49] Herbig J, Reimann C T, Bergquist M, Oscarsson S and Vorobyova I V 1999 Nucl. Instrum. Methods B 149119

[50] Trautmann C, Bocanfuso M, Benyagoub A, Klaumünzer S, Schwartz K and Toulemonde M 2002 Nucl. Instrum. Methods Phys. Res. B 191144

[51] Sattonnay G, Lahrichi M, Herbst-Ghysel M, Garrido F and Thomé L 2007 J. Appl. Phys. 101103516

[52] Bianconi M, Bentini G G, Chiarini M, de Nicola P, Montanari G B, Nubile A and Sugliani S 2009 Nucl. Instrum. Methods Phys. Res. B 2672839

[53] Rebouta L, Smulders P J M, Boerma D O, Agulló-López F, da Silva M F and Soares J C 1993 Phys. Rev. B 483600

[54] Skuratov V A, Zinkle S J, Efimov A E and Havancsak K 2003 Nucl. Instrum. Methods Phys. Res. B 203136

[55] Trautmann C, Toulemonde M, Constantini J M, Grob J J and Schwartz K 2000 Phys. Rev. B 6213

[56] Saleh S A and Eyal Y 2005 Nucl. Instrum. Methods Phys. Res. B 23681

[57] Moreira P A F, Devanathan R and Weber W J 2010 J. Phys. C: Condens. Matter 22395008

[58] Rivera A, Crespillo M L, Olivares J, García G and Agulló-López F 2010 Nucl. Instrum. Methods Phys. Res. B 2682249

[59] García G, Rivera A, Crespillo M L, Gordillo N, Olivares J and Agulló-López F 2011 Nucl. Instrum. Methods Phys. Res. B 269492

[60] Wong K S (ed) 1989 Properties of Lithium Niobate (EMIS Datareviews Series No. 5) INSPEC

[61] Ramos S M M, Brenier R, Canut B, Fuchs G, Thevenard P, Trilleux M, Meftah A and Toulemonde M 1995 J. Appl. Phys. 772952

[62] Götz G and Karge H 1983 Nucl. Instrum. Methods 209/210 1079

[63] Bazzan M 2007 private communication, University of Padova

[64] Fries E and Péter A 1987 Rev. Phys. Appl. 221353 